\title{
Beam Loading Issues for SNS Storage Ring*
}

\author{
W.T. Weng and S.Y. Zhang \\ AGS Department, Brookhaven National Laboratory, Upton, NY, USA
}

Introduction

In the Spallation Neutron Source (SNS) storage ring, the machine cycle is dominated by the multiturn injection. Therefore, the beam loading issues are quite different from the conventional beam loading. The evolution of the particle distribution due to beam loading needs attentions. If not corrected, the beam loading effect will lead to a bunch to bucket mismatch in the injection, causing bunch leakage into the interbunch gap. The bunch leakage will increase the extraction beam loss, and lower the e-p instability threshold On the other hand, the control of bunch leakage necessitates the beam loading factor to be small. Therefore, beam stabilities associated with the beam loading automatically satisfied. In this article, the simulation of the SNS beam loading effect (together with the space charge effect) will be presented. Same method has been applied to the PSR, where some beam loading effect is shown.

\section{Beam Loading}

For multiturn injection at the SNS, the beam intensity increases gradually within a period of 1,200 turns of injection, each turn takes $841 \mathrm{~ns}$. The loaded RF cavity, therefore, sees a bunch train with a fixed revolution frequency and a constantly increasing intensity during the injection.

The impedance of the loaded cavity can be represented as,

$$
Z=\frac{2 \sigma R s}{s^{2}+2 \sigma s+\omega_{R}^{2}}
$$

where $s$ is the Laplace factor, $R$ is the resistance of the loaded cavity, ferrite and generator in parallel, $\omega_{R}$ is the resonant frequency, which equals to the revolution frequency $\omega_{0}$ at the SNS. The time constant $\tau$ of the cavity voltage response, with respect to the incoming bunch train, is determined by the cavity half bandwidth $\sigma$ as

$$
\tau=\frac{1}{\sigma}
$$

\footnotetext{
* Work performed under the auspices of the US Dept. of Energy
}

with

$$
\sigma=\frac{1}{2 R C}
$$

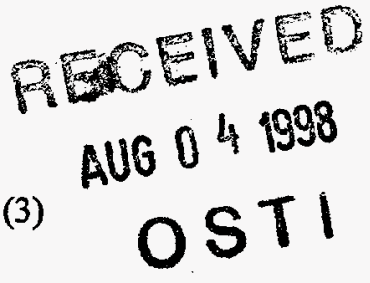

where $C$ is the cavity capacitance.

Usually, the beam loading effect is judged by the beam loading factor, which is defined as,

$$
Y=\frac{I_{B}}{I_{G}}
$$

where $I_{G}$ is the generator current. For the SNS, with the beam loading factor $Y>1$, the bunch leakage would become unacceptable. Also under this condition, other remedies to control the bunch leakage, such as cavity detuning, become unreliable. Therefore, we only consider the situation that $Y<1$. Thus, the beam loading effect we consider is not the conventional one, which addresses beam instabilities. Instead, the beam loading effect at the SNS addresses the evolution of the particle distribution, in particular, the possibility of bunch leakage into the interbunch gap.

\section{Simulation}

The SNS parameters used for the simulations are as follows. The kinetic energy $E_{k}=1 \mathrm{GeV}$, circumference $C=220.69 \mathrm{~m}$, revolution frequency $f_{0}=1.189 \mathrm{MHz}$ are used. The machine average vacuum chamber radius $b=10$ $\mathrm{cm}$ and the beam size $a=2.36 \mathrm{~cm}$ give rise to the longitudinal space charge impedance $Z_{s c}=-j 196 \Omega$. Note that the beam size $a=\sqrt{2} \sigma$, where $\sigma$ is the rms size.

The incoming Linac beam is chopped at the width of $\pm 0.67 \pi$, and the momentum spread is somewhat $d p / p= \pm 0.2 \%$. The $\mathrm{RF}$ voltage is $V_{R F}=40 \mathrm{KV}$ per turn, with a second harmonic system of half of that voltage. The injection with 1,200 turns results in $N=2 \times 10^{14}$ per pulse.

For the particle longitudinal motion, the following equations are used in the simulation,

$$
\frac{d}{d t}\left(\frac{\Delta E}{\omega_{0}}\right)=\frac{e}{2 \pi}\left(V_{R F}(\sin \phi-0.5 \sin 2 \phi)+V_{S C}+V_{B L}\right)
$$

and

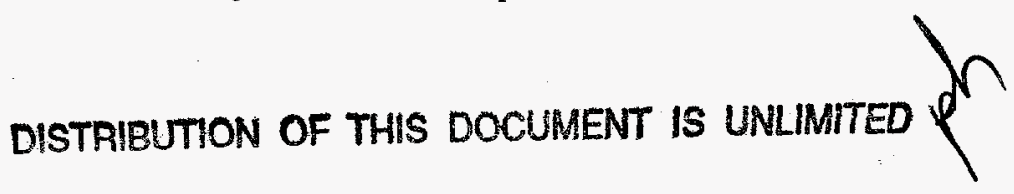

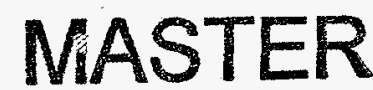




\section{DISCLAIMER}

This report was prepared as an account of work sponsored by an agency of the United States Government. Neither the United States Government nor any agency thereof, nor any of their employees, makes any warranty, express or implied, or assumes any legal liability or responsibility for the accuracy, completeness, or usefulness of any information, apparatus, product, or process disclosed, or represents that its use would not infringe privately owned rights. Reference herein to any specific commercial product, process, or service by trade name, trademark, manufacturer, or otherwise does not necessarily constitute or imply its endorsement, recommendation, or favoring by the United States Government or any agency thereof. The views and opinions of authors expressed herein do not necessarily, state or reflect those of the United States Government or any agency thereof. 


\section{DISCLAIMER}

Portions of this document may be illegible electronic image products. Images are produced from the best available original document. 


$$
\frac{d}{d t} \phi=\frac{\omega_{0}^{2} \eta}{\beta^{2} E}\left(\frac{\Delta E}{\omega_{0}}\right)
$$

where $\Delta E$ is the energy gain per turn, $\phi$ is the beam phase deviation in radius, $V_{S C}$ and $V_{B L}$ represent space charge and beam loading voltages, respectively.

For each turn of injection, the space charge voltage is calculated using the beam line density $I$, as [1],

$$
V_{S C}=\frac{d I}{d \phi}\left|Z_{S C}\right|
$$

The SNS RF system and its feedback are currently under study [2]. The purpose of this article is to look at the beam loading induced bunch leakage. Therefore, we simply assume that the resistance of the loaded cavity (with, say, one turn feedback) to be $400 \Omega, 200 \Omega$, and $100 \Omega$ per ring, together with a capacitance $500 \mathrm{pf}$.

For each bunch passage, the loaded cavity sees a beam current, and it is responded with a beam loading voltage $V_{B L}$ on the cavity. This voltage has the fundamental mode, and it is out of phase with the RF voltage by about 90 degrees.

If we take $R=400 \Omega$, and $C=500 p f$, it is found $\tau=400 \mathrm{~ns}$, which is well below the beam circulating period of $841 \mathrm{~ns}$. Since the intensity of the beam increases gradually and the cavity time constant is small, the beam loading voltage increases in proportional to the beam intensity. Thus, the transient effect of the beam loading can be neglected, and the static beam loading can be assumed.
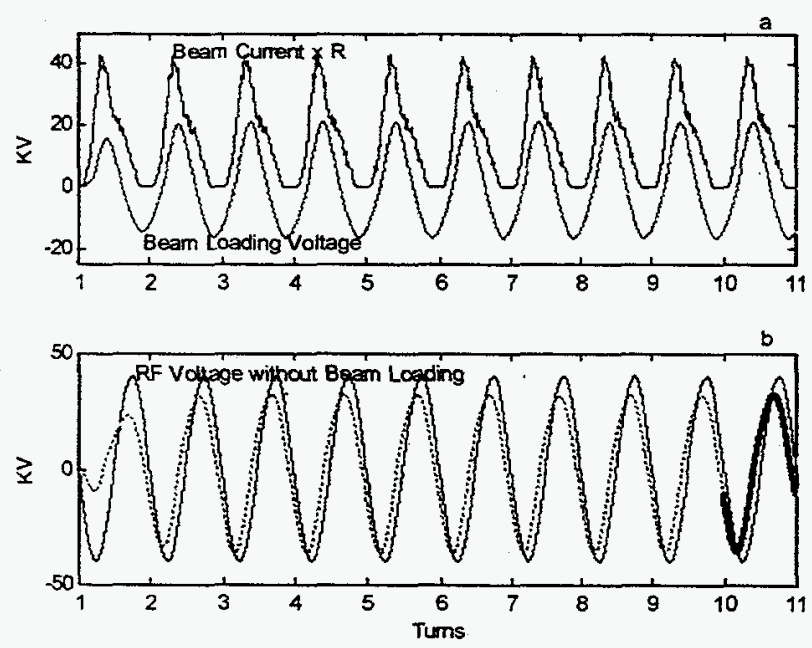

Fig.1. Beam Loading Voltage used in Simulation

In the simulation, for each turn, 10 sequential beam line densities are stored and used as input to the loaded cavity, thus to get the beam loading voltage. One example is shown in Fig.1. The (false) transient response is only shown up in first couple of turns in Fig.1. Therefore, we use the response of the last turn (10th turn) as the beam loading voltage for this turn, which is marked by bold line in Fig. 1 .

For the detuned cavity, similar technique is used to get the effective RF voltage. Both the RF signal $(h=1)$ and the beam line density are used as input to the detuned cavity, then the cavity response in the 10th turn is used in the simulation. The second harmonic is not changed in the simulation.

Total 12,000 macroparticles are used, the time step used is one turn. The machine circumference is split into 30 bins in calculating the space charge induced voltage. To split into 60 and 120 bins did not show visible improvement in the simulations. In calculating the beam line density, minor smoothing has been used.

\section{Results}

In the first set of simulations, a fixed RF voltage of $40 \mathrm{KV}$ (20 KV for 2nd harmonic) is used. Cavity detuning angle is set to zero, and the effective resistance of the loaded cavity is $400 \Omega, 200 \Omega$, and $100 \Omega$. Resulted particle distributions and the associated beam line densities at the end of injection are shown in Fig. $2 \mathrm{a}, 2 \mathrm{~b}$, and $2 \mathrm{c}$, respectively.
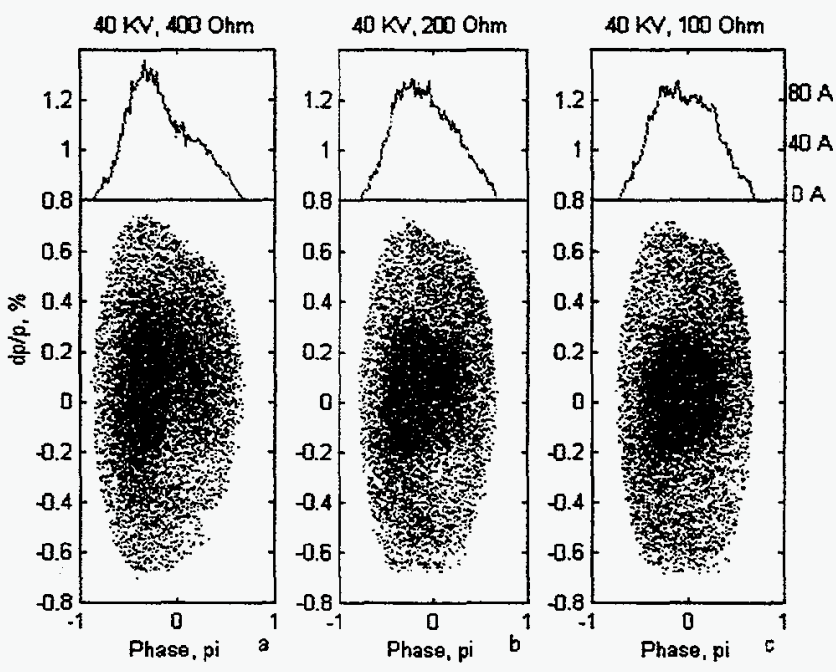

Fig.2. SNS Beam Loading Effect with $V_{R F}=40 K V$

Because of the beam loading induced phase shift of the effective RF voltage, the left end of the bunch length is $0.87 \pi, 0.78 \pi$, and $0.74 \pi$, for the cases $a, b$, and $c$, whereas the other end is the same as the injected beam, $0.67 \pi$. We also note that all beam momentum spread in the end of injection is about $0.70 \%$.

One way to limit the bunch leakage is to use the RF voltage ramping during the injection. For instance, to linearly increase the $h=1$ cavity voltage from $20 \mathrm{KV}$ at the start to $40 \mathrm{KV}$ at the end of injection. In this way, the beam momentum spread in ring, defined during the multiturn injection, can be lowered. Hence, the longitudinal space charge induced bunch lengthening can be reduced.

In Fig. $3 a, 3 b$, and $3 c$, the counterparts to the cases shown in Fig.2 are shown, with the RF voltage ramping 
from $20 K V$ to $40 K V$ (2nd harmonic RF voltage as half of that voltage). The left end of the bunch length is $0.86 \pi, 0.73 \pi$, and $0.68 \pi$, for the cases $3 a, 3 b$, and $3 c$, respectively. Meanwhile, all the beam momentum spread at the end of injection is about $0.63 \%$.
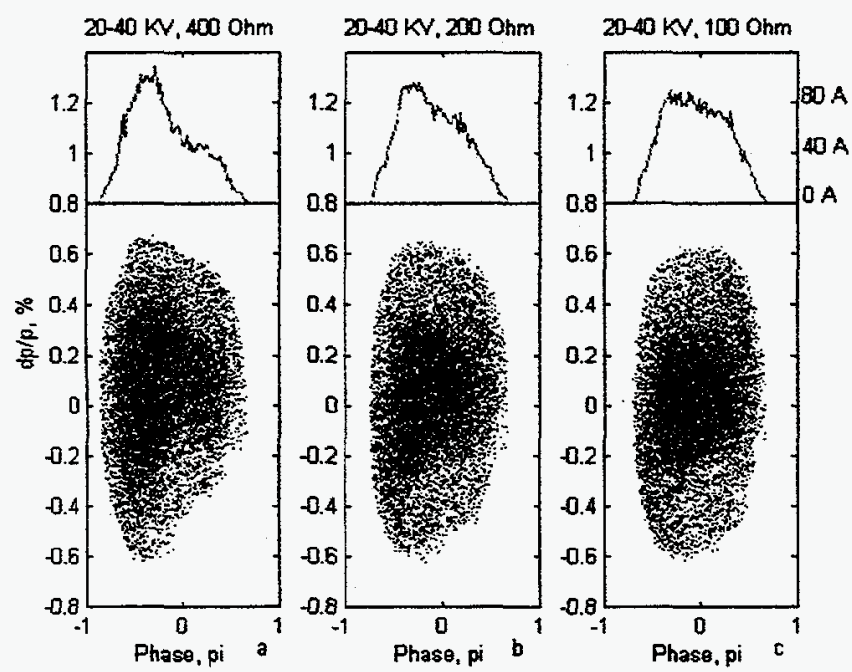

Fig.3. SNS Beam Loading Effect with Ramping RF Voltage

The bunch length of cases $2 \mathrm{a}$ and $3 \mathrm{a}$ are almost identical, showing that at $R=400 \Omega$, the space charge effect is overwhelmed by beam loading effect. The bunch length in case $3 b$ has been reduced considerably from that in case $2 b$. As for case $3 c$, the bunch lengthening is essentially eliminated.

\section{Discussion}

1. Simulation results have shown that if the resistance of loaded cavity is $R=200 \Omega$ (with feedback applied) per ring, then using the RF voltage ramping during the injection leads to a moderate bunch leakage. At $R=400 \Omega$, the bunch leakage becomes unacceptable.

2. Dynamic cavity detuning in a time period of $1 \mathrm{~ms}$ is difficult. Also in SNS, the detuning range needed is large, for instance, at $R=400 \Omega$ and $N=2 \times 10^{14}$, the cavity detuning frequency would be $220 \mathrm{KHz}$ at the end of the injection. On the other hand, the cavity detuning could be preset to partially compensate the beam loading effect. In Fig.4, an example with a preset cavity detuning 20 degree from the case $3 a$ are shown. Improvements can be observed. However, the irregular bunch shape, especially the shifting of the bunch mass center during the multiturn injection would make the feedback very difficult.

3. The mismatch of injected beam to the feedback regulated bucket will likely cause particle spread in longitudinal space. Barrier cavities with relatively small power may help to stop these particles to get into the gap.

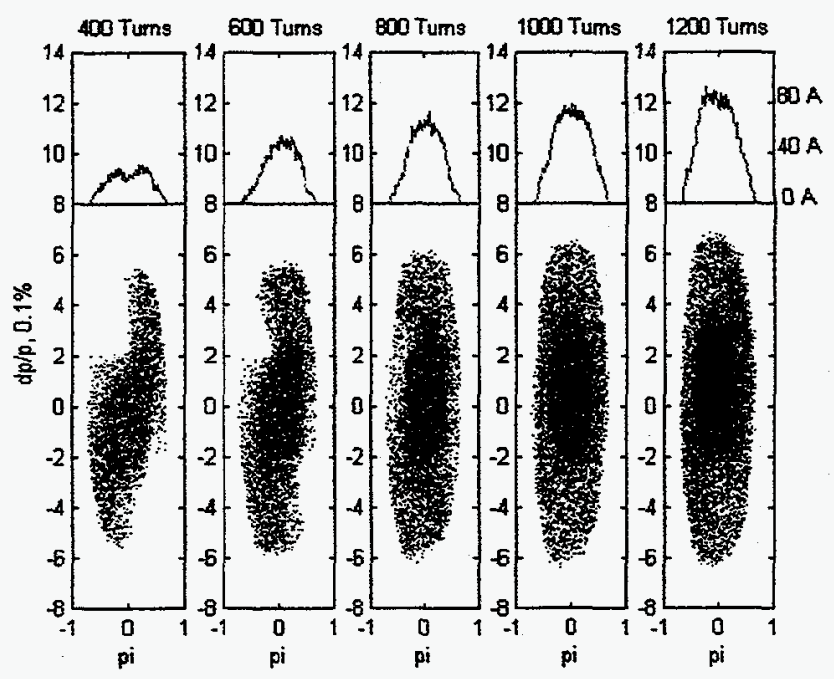

Fig.4. SNS Beam Loading Effect with Cavity Detuning

4. Same simulation method has been applied to the PSR [3], and it is shown that for $R=120 \Omega$ per ring, without cavity detuning, the beam loading caused bunch leakage is larger than the space charge effect. This is shown in Fig.5. The longitudinal space charge impedance of PSR is similar to that of SNS. The beam intensity is $N=3 \times 10^{13}$, however, the bunch is only $250 \mathrm{~ns}$, vs. $550 \mathrm{~ns}$ of SNS. Thus, the space charge voltage is comparable with the SNS. On the other hand, the $\mathrm{RF}$ voltage there is from $6 \mathrm{KV}$ to $10 \mathrm{KV}$.
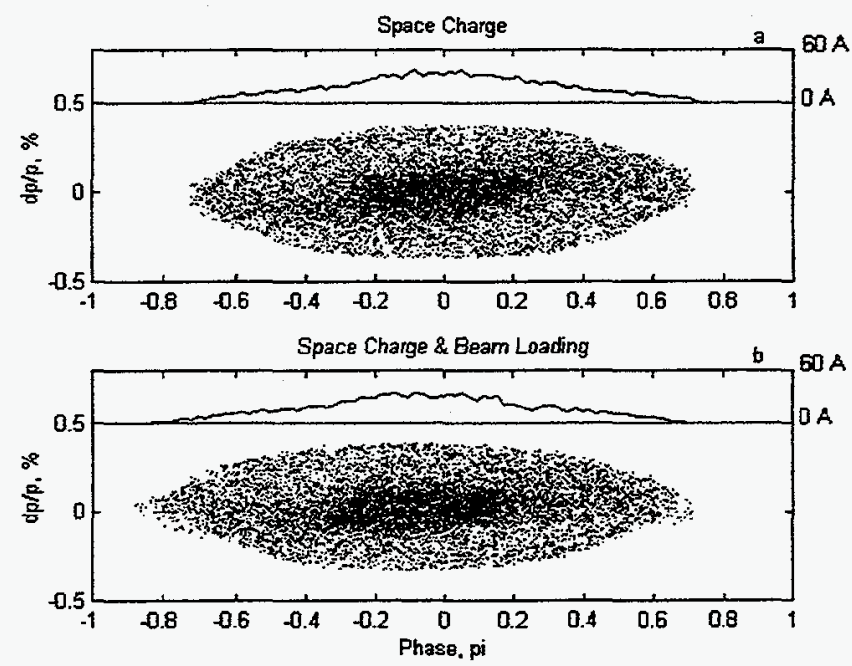

Fig.5. PSR Beam Loading Effect

\section{Reference}

1. S.Y. Zhang and W.T. Weng, these proceedings.

2. M. Blaskiewicz, J.M. Brennan, and A. Zaltsman, BNL/SNS Tech. Note. No.36, May, 1997.

3. S.Y. Zhang and W.T. Weng, 'Beam Loading Effect in Bunch Leakage at the PSR', Nucl. Instrum. Methods, in press. 